\title{
Human-Network Regions as Effective Geographic Units for Disease Mitigation
}

\author{
Clio Andris ${ }^{1}$, Caglar Koylu ${ }^{2}$, and Mason A. Porter ${ }^{3}$ \\ ${ }^{1}$ School of City and Regional Planning, School of Interactive Computing, Georgia Institute of Technology \\ ${ }^{2}$ Geographical and Sustainability Sciences, University of Iowa \\ ${ }^{3}$ Department of Mathematics, University of California, Los Angeles and Santa Fe Institute
}

October 15, 2021

\begin{abstract}
Susceptibility to infectious diseases such as COVID-19 depends on how they spread, and many studies have captured the decrease in COVID-19 spread due to reduction in travel. However, less is known about practical geographic boundaries for that limit the spread of COVID-19 to adjacent places. To detect such boundaries, we apply community-detection algorithms to large networks of mobility and social-media connections to construct geographic regions that reflect natural human movement and relationships at the county level for the continental United States. We measure COVID-19 cases, case rates, and case-rate variations across adjacent counties and examine how often COVID-19 crosses the boundaries of these functional regions. We find that regions that we construct using GPS-trace networks and especially commuter networks have the smallest rates of COVID-19 case rates along the boundaries, so these regions may reflect natural partitions in COVID-19 transmission. Conversely, regions that we construct from geolocated Facebook friendships and Twitter connections yield the least effective partitions. Our analysis reveals that regions that are derived from movement flows are more appropriate geographic units than states for making policy decisions about opening areas for activity, assessing vulnerability of populations, and allocating resources. Our insights are also relevant for policy decisions and public messaging in future emergency situations.
\end{abstract}

Keywords: COVID-19, Network Modularity, Geographic Regions, Social Networks

SIGNIFICANCE STATEMENT: We construct regions for the continental United States that follow natural principles of human movement, migration, and social-network connections. In comparison to state units, large numbers of COVID-19 cases and large case rates proliferate within regions and are smaller across regions, especially ones that we build from GPS trace and commuting networks. These regions can serve as effective geographic units for implementing stay-at-home orders, delivering public messaging, and capturing cohesive areas with shared movement and social networks. This finding is also relevant for policy decisions in future pandemics. 


\section{Introduction}

Coronavirus disease 2019 (COVID-19) has caused over 700,000 deaths and more than 40 million infections in the United States and over 4.5 million deaths and more than 200 million cases worldwide [8]. Although new vaccines help mitigate the harmful effects of the disease, in 2020 and early in 2021, non-pharmaceutical interventions (NPIs) were the primary method of protecting individuals from exposure to severe acute respiratory syndrome coronavirus 2 (SARS-COV-2), which causes COVID-19. These interventions have included formal shelter-in-place rules and guidelines, facility closures, limited seating in restaurants, reduction of interactions through physical distancing (which is often called 'social distancing'), and travel-restriction policies to reduce mobility and transmission [10]. Such NPIs have generally been effective [35].

In the United States, policies that invoke NPIs are typically administered at the state level. This has resulted in friction in local communities that seek to impose different (and often more stringent) standards than other areas in their state $[26,16,1]$. To avoid spillovers of COVID-19, people should stay within a local region for daily activities $[43,25]$. However, when this region (e.g., a metropolitan area) naturally spans across multiple states or is experiencing different risk levels than the rest of a state, it may be subject to conflicting policies. To define units for which it is reasonable to apply homogeneous NPI policies, we seek to construct regions that capture core geographies of social and movement behavior. When using these regions as administrative units, there is likely to be less inter-regional movement that can exacerbate the spread of COVID-19 [42, 50] than when using states.

The objective of defining regions that may not follow administrative boundaries is not new, but it has deep theoretical roots in regional science, economic geography, and human geography [36, 37, 30]. Defining regions that are based on news markets, vacation trips, telecommunications, $[21,34,18,7]$ and (more recently) commuters, GPS traces, flights, migration, and social-media relationships [36, 20, 27, 32, 6, 31] has been a common practice for decades [18, 13, 24], but such regions are rarely implemented in practice for policy purposes. Recently, however, [27] divided two Wisconsin counties into regions using the Walktrap community detection algorithm on SafeGraph mobility data; these regions served as effective boundaries for COVID-19 transmission, wherein about half of infections occurred within regions [27].

To assess the ability of such regions to capture cohesive areas with high COVID-19 rates, it is desirable to know the transmission patterns of SARS-CoV-2. However, modeling the transmission of COVID-19 infections in networks of individuals is complicated by asymptomatic transmissions and other factors [3]. Phylogenetic strains of SARS-CoV-2 indicate that the virus's subsequent mutations, such as the Delta variant, initially tended to stay within concentrated geographic regions [23], thus hinting at the geographic spread of the virus. However, as mutated variants of SARS-CoV-2 propagated, geographic transmission paths became too widespread to pinpoint.

Accordingly, contact-tracing technologies that record geographic traces of infected individuals [14] have had mixed results because of underdeveloped technologies, mixed participation levels from individuals, and lack of administrative organization and oversight [26]. Despite a lack of information on the precise spatial transmission of SARS-CoV-2, we can assess how different sets of regional boundaries act as informal barriers to disease transmission. We posit that regions that one obtains from human behavior may help explain the spatiotemporal landscape of COVID-19 case rates (as in $[27])$. 
We investigate the extent to which regional boundaries that are based on five county-level networks in the lower 48 U.S. States (commuters, GPS-based trips, migration, Twitter connections, and Facebook connections) capture smaller COVID-19 case rates and fewer cases than state boundaries.

We expect to observe large case rates within these functional geographic regions, with low transmission activity (which we measure by total cases per capita) between regional boundaries. We also investigate whether case rates are more homogeneous within regions than between regions. Because cohesive metropolitan areas often straddle borders, we posit that the regions that we construct from human-mobility dynamics will more effectively capture natural bottlenecks in a disease-transmission network than social-media-based regions or administrative boundaries such as states.

Some networks have firmer theoretical grounding than others for the problem at hand. For example, commutes and GPS traces directly indicate movement, whereas social-media networks encode proclivities to spread information; the latter may be less appropriate for examining or explaining disease transmission. However, because social-media relationships tend to follow networks of movement [45], data from them may still provide a heuristic indicator of appropriate boundaries.

By determining functional regional units for the management of the spatial transmission of a disease (and perhaps particularly so during a pandemic), we suggest flexible alternatives to using states as administrative units for policy implementation. Because these proposed alternatives are based on human behavior, they can help limit disease transmission while permitting some natural activity (such as social visits and travel).

\section{Results}

\subsection{Constructed Regions}

We use the Louvain method of maximizing the 'modularity' objective function [5] to detect communities and create regions from our five human behavior-based networks (see Materials and Methods). Of the five networks, the commuter network yields the most geographic regions (with $n(r)=75$ regions), and the Twitter network and migration networks yield the fewest (26 and 28 regions, respectively). See Table 1 and Figure 1. The commuter network and GPS-trip network result in the largest values of maximized modularity $Q_{\max }$ (which indicates the amount of compartmentalization in a network $[39,15])$. However, these networks have the most regions.

Table 1: Basic statistics of the constructed regions. We give the number $n(r)$ of geographic regions, the maximized modularity $Q_{\max }$, the total distance $d$ of the boundaries, the number $E_{\mathrm{b}}$ of edges between regions, the number $E_{\mathrm{w}}$ of edges within regions, and $d / E_{\mathrm{b}}$.

\begin{tabular}{lrrrrrr}
\hline Region Type & $n(r)$ & $Q_{\max }$ & $d($ in $\mathrm{km})$ & $E_{\mathrm{b}}$ & $E_{\mathrm{w}}$ & $d / E_{b}$ \\
\hline States & 48 & n/a & 36,400 & 1,082 & 7,068 & 26.86 \\
Commuters & 75 & 0.95 & 55,501 & 1,504 & 6,646 & 30.95 \\
Facebook & 33 & 0.87 & 33,587 & 1,001 & 7,149 & 28.51 \\
Migration & 28 & 0.80 & 30,413 & 887 & 7,263 & 28.40 \\
Trips & 53 & 0.91 & 43,282 & 1,218 & 6,932 & 30.12 \\
Twitter & 26 & 0.74 & 29,674 & 848 & 7,302 & 28.73 \\
\hline
\end{tabular}

To model COVID-19 cases, case rates, and case-rate differences, we use a network $G_{a}$ of county adjacencies with 3,108 county centroids as the nodes. There is an undirected edge between two nodes whenever their associated counties share a border. Each county had at least one COVID-19 


\section{STATES (48)}

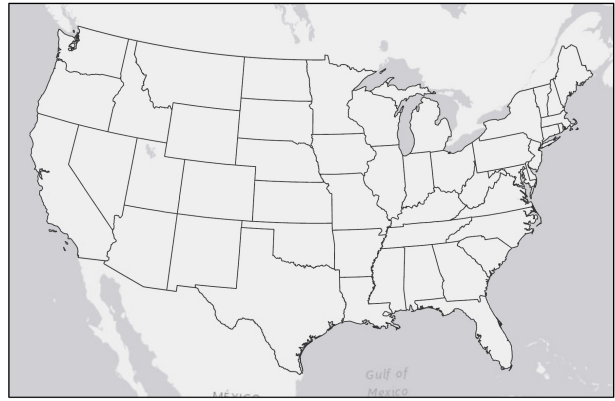

FACEBOOK REGIONS (33)

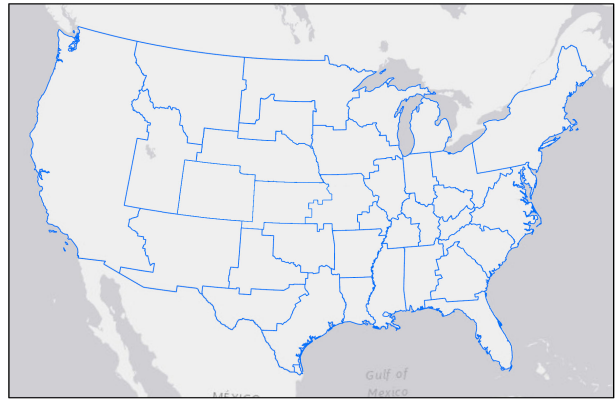

TRIPS REGIONS (53)

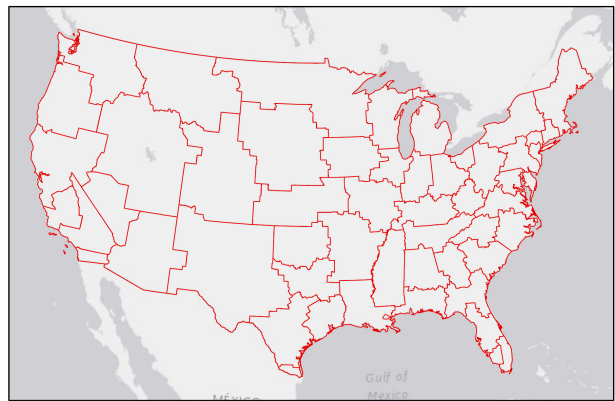

\section{COMMUTER REGIONS (75)}

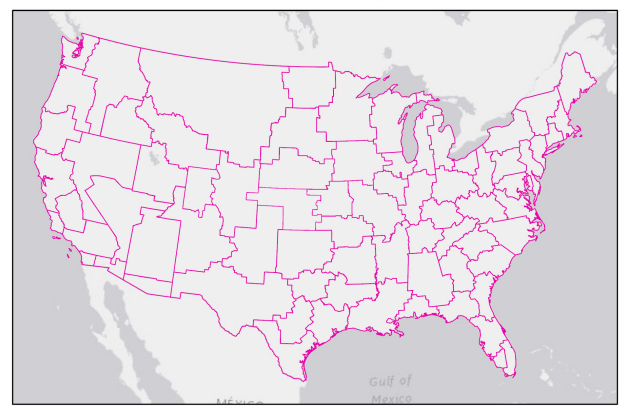

MIGRATION REGIONS (28)

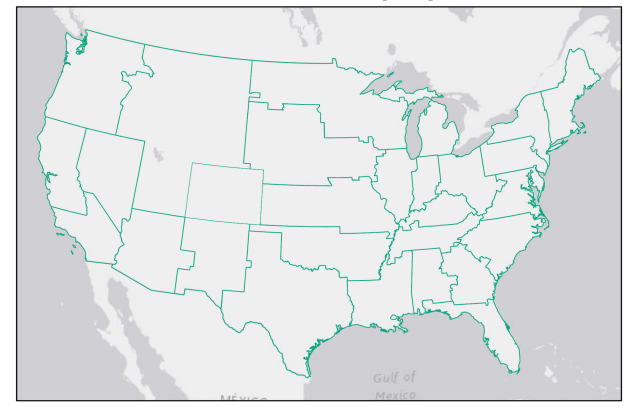

TWITTER REGIONS (26)

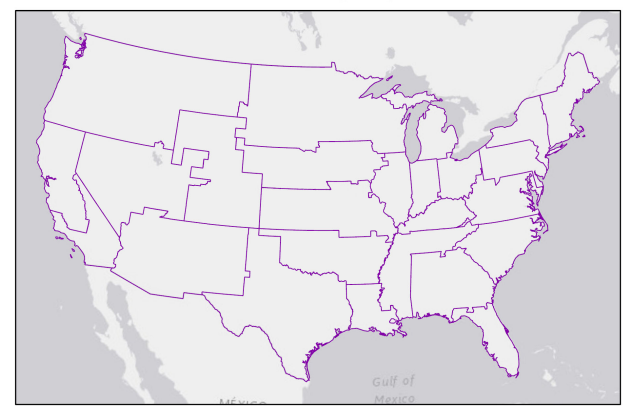

Figure 1: State boundaries and five regional boundaries in the United States. We algorithmically detect the regional boundaries from human-behavior networks using the Louvain method of modularity maximization [5]. We show the number of resulting regions in parentheses.

case as of 31 May 2020; this yields 8,150 edges. We denote the total number of edges that traverse two regions by $E_{\mathbf{b}}$, and we denote the the total number of edges that remain within a region by $E_{\mathrm{w}}$. (The sum of $E_{\mathrm{b}}$ and $E_{\mathrm{w}}$ is 8,150.) Because the geometry (specifically, the area and shape) of the regions and the numbers $n(r)$ of regions are different in each network, some sets of regions provide more opportunities for crossings. The number $n(r)$ of regions correlates both with the length $d$ of the internal boundaries and with the number $E_{\mathrm{b}}$ of between-region crossings. The Pearson product-moment correlation coefficients are $f\left(E_{b}, d\right) \approx 0.986, f\left(E_{b}, n(r)\right) \approx 0.999$, and $f(d, n(r)) \approx 0.997$. We use $d / E_{b}$ to denote the number of between-region crossings per kilometer of border, and we find that $d / E_{b}$ is roughly 30 kilometers (see Table 1 ). 


\subsection{COVID-19 Cases Between and Within Regions}

We discuss cases rates on edges (which we denote by $\mathrm{CR}_{b}$ for between-region edges and by $\mathrm{CR}_{w}$ for within-region edges) and the differences of case rates (which we denote by $\mathrm{CD}_{b}$ for between-region edges and by $\mathrm{CD}_{w}$ for within-region edges) on an edge. We report case rates as cases per 1,000 individuals (see Materials and Methods).
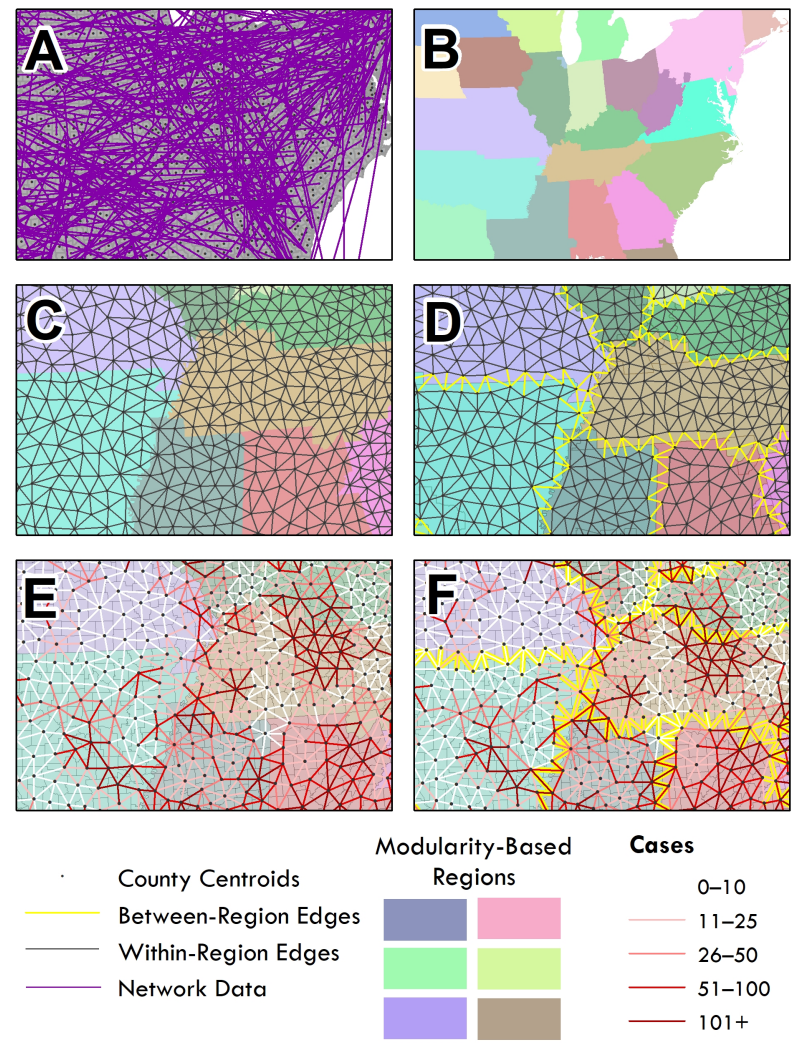

Figure 2: Each network-partitioning method has an input of (A) a county-to-county network of movement flows or social-media connections. We apply a community-detection algorithm to create (B) distinct regions. We use (C) a network $G_{a}$ of county adjacencies and (D) distinguish edges between regions ( $E_{\mathrm{b}}$, in yellow) from those within regions $\left(E_{\mathrm{w}}\right.$, in black). (E) We then weight all edges $E$ by COVID-19 cases, case rates, and differences of case rates. (F) We measure these values both between regions (in yellow) and within regions.

\subsubsection{Region-type variation in case rates and case-rate differences}

We first measure COVID-19 case rates between and within regions (see Figure 2); we obtain the same qualitative results for all three temporal waves of data (see Figure 2). The case rates within regions $\left(\mathrm{CR}_{w}\right)$ are largest for commuter and trip regions, and they are smallest for the Facebookbased regions (see Table 2). The case rates between regions $\left(\mathrm{CR}_{b}\right)$ are smallest for the commute and trip regions (indicating a low penetration of cases per capita across the boundaries) and are largest for the Facebook and migration regions.

The case-rate differences within regions $\left(\mathrm{CD}_{w}\right)$ are smallest for commutes and second smallest for states (Table 2), indicating that there are similar case rates in counties that are in the same region 
for these two types of divisions. In terms of $\mathrm{CD}_{b}$, where larger values indicate more case-rate heterogeneity, we find that regions derived from migration and states, followed by commutes, most effectively mark divisions in case rates. Those derived from Facebook and trips serve as the least effective partitions in this regard. These calculations present the view that large differences in rates across states may suggest that states are more effective partitions than we initially posited. However, the regions have different performances for different temporal waves.

Table 2: Median values of COVID-19 case rates $\left(\mathrm{CR}_{b}\right.$ and $\left.\mathrm{CR}_{w}\right)$, case-rate differences $\left(\mathrm{CD}_{b}\right.$ and $\left.\mathrm{CD}_{w}\right)$, and cases $\left(C_{b}\right.$ and $\left.C_{w}\right)$ between and within regions.

\begin{tabular}{lccccccr}
\hline Region Type & $\mathrm{CR}_{w}^{*}$ & $\mathrm{CR}_{b} *$ & $\mathrm{CD}_{w}$ & $\mathrm{CD}_{b}$ & $\frac{\left(C_{b} / C_{w}\right)}{\left(E_{b} / E_{w}\right)}$ & $C_{w}$ & $C_{b}$ \\
\hline Wave 1 (21 January 2020 - 31 May & $2020)$ & & & & & \\
\hline Commutes & 1.75 & 1.48 & 0.98 & 0.96 & 2.22 & 143 & 72 \\
Facebook & 1.71 & 1.60 & 0.96 & 1.07 & 6.02 & 127 & 107 \\
Migration & 1.70 & 1.72 & 0.96 & 1.07 & 5.46 & 129 & 86 \\
States & 1.69 & 1.73 & 0.95 & 1.11 & 5.60 & 127 & 109 \\
Trips & 1.71 & 1.63 & 0.98 & 0.96 & 3.35 & 135 & 80 \\
Twitter & 1.69 & 1.77 & 0.95 & 1.18 & 6.63 & 128 & 99 \\
\hline Wave 2 (1 June 2020 -31 August 2020$)$ & & & & & \\
\hline Commutes & 9.30 & 7.63 & 3.24 & 3.52 & 2.43 & 727 & 400 \\
Facebook & 8.88 & 9.86 & 3.19 & 4.19 & 8.24 & 630 & 727 \\
Migration & 9.03 & 8.85 & 3.23 & 4.00 & 6.10 & 663 & 494 \\
States & 9.08 & 8.68 & 3.26 & 3.60 & 5.99 & 642 & 589 \\
Trips & 9.15 & 8.11 & 3.25 & 3.65 & 3.61 & 695 & 441 \\
Twitter & 9.08 & 8.46 & 3.24 & 3.83 & 6.79 & 656 & 517 \\
\hline Wave 3 (1 September 2020 & $1 \mathrm{July}$ & $2021)$ & & & & & \\
\hline Commutes & 91.10 & 89.14 & 12.66 & 15.37 & 2.89 & 6140 & 4011 \\
Facebook & 90.42 & 93.16 & 12.91 & 14.78 & 7.43 & 5600 & 5829 \\
Migration & 90.61 & 91.51 & 12.80 & 15.71 & 6.39 & 5796 & 4524 \\
States & 90.75 & 90.97 & 12.73 & 15.51 & 6.36 & 5662 & 5513 \\
Trips & 90.96 & 89.54 & 12.83 & 14.82 & 3.90 & 5995 & 4112 \\
Twitter & 90.74 & 91.08 & 12.85 & 15.21 & 7.48 & 5728 & 4975 \\
\hline
\end{tabular}

* Case rates per 1,000 individuals.

\subsubsection{Raw case odds ratios}

The total number of COVID-19 cases on an edge (which we denote by $\mathrm{C}_{b}$ for between-region edges and by $\mathrm{C}_{w}$ for within-region edges) is sensitive to the number of potential crossings across regions. Nevertheless, we report the odds ratio $\frac{\mathrm{C}_{b} / \mathrm{C}_{w}}{\mathrm{E}_{b} / \mathrm{E}_{w}}$ of cases to edges to estimate the ratio of cases between regions to cases within regions. This ratio is largest in the Facebook regions and second largest in the Twitter regions, providing further evidence that these partitions are the least effective of the six that we examine. By contrast, commuter and trip regions have the smallest ratios and thus perform the best (see Table 2). 


\subsubsection{Statistical tests}

We now test for statistical significance in the COVID-19 indicators: cases, case rates, and case-rate differences at the level of edges using data from wave 3, which is the most recent wave. Using an ANOVA test, we obtain an F statistic for cases between regions $\left(\mathrm{C}_{b}\right)$ of 1.850 (with a probability of more than $\mathrm{F}$ of 0.0996 ) and an $\mathrm{F}$ statistic for $\mathrm{CR}_{b}$ of 5.457 (with a probability of more than $\mathrm{F}$ of $5.13 \times 10^{-5}$ ) (Table 3). No other value is significant. This result suggests that the differences among the six region types are best distinguished by COVID-19 case-rate differences on their borders, rather than such differences within their regions.

In our permutation analysis, we find that the COVID-19 indicators on between-region edges and within-region edges differ significantly from each other. The variation is most pronounced for regions that we derived from commutes, followed by those from trip and migration regions (Table 4). The case-rate differences (CD) reveal the strongest distinctions between between-region edges and within-region edges. These results illustrate that movement data best 'carve' around COVID-19 indicators and that this process is best captured by measuring case-rate differences CD.

Table 3: Results of ANOVA tests to find significant variation across region types for Wave 3

\begin{tabular}{|c|c|c|c|c|c|}
\hline Region Type & $\mathrm{DF}$ & Sum of Squares & Mean Sq. & F Value & $\operatorname{Pr}(>\mathrm{F})$ \\
\hline \multicolumn{6}{|l|}{ Within-Region Results } \\
\hline Cases $\left(\mathrm{C}_{w}\right)$ & 5 & $3.792 \times 10^{9}$ & $7.583 \times 10^{8}$ & 0.385 & 0.859 \\
\hline Case Rates $\left(\mathrm{CR}_{w}\right)$ & 5 & 1,670 & 334 & 0.706 & 0.619 \\
\hline Case-Rate Differences $\left(\mathrm{CD}_{w}\right)$ & 5 & 666 & 133.2 & 0.354 & 0.880 \\
\hline \multicolumn{6}{|l|}{ Between-Region Results } \\
\hline Cases $\left(\mathrm{C}_{b}\right)$ & 5 & $1.707 \times 10^{10}$ & $3.413 \times 10^{9}$ & 1.850 & 0.0996 . \\
\hline Case Rates $\left(\mathrm{CR}_{b}\right)$ & 5 & 10,067 & $2,013.5$ & 5.457 & $5.13 \times 10^{-5} * * *$ \\
\hline Case-Rate Differences $\left(\mathrm{CD}_{b}\right)$ & 5 & 2,108 & 421.6 & 1.288 & 0.266 \\
\hline
\end{tabular}

Significance levels: '***) $0.001 ;{ }^{\text {(**) }} 0.01$; $^{* *}$ ' 0.05 ; and '.' 0.1 .

Table 4: Results of permutation tests across region types for Wave 3

\begin{tabular}{|c|c|c|c|}
\hline Region Type & $\begin{array}{r}\text { COVID-19 Cases }(\mathrm{C}) \\
(\mathrm{P}>\text { Mean })\end{array}$ & $\begin{array}{r}\text { COVID-19 Rates }(\mathrm{CR}) \\
(\mathrm{P}>\text { Mean })\end{array}$ & $\begin{array}{r}\text { Rate Difference }(\mathrm{CD}) \\
(\mathrm{P}<\mathrm{Mean})\end{array}$ \\
\hline Commutes & $0 * * *$ & $0.0045^{* *}$ & $1 * * *$ \\
\hline Facebook & 0.3499 & 0.9999 & 0.8829 \\
\hline Migration & $0 * * *$ & 0.8912 & $0.9996 * *$ \\
\hline States & 0.1957 & 0.216 & $0.9999 * * *$ \\
\hline Trips & $0 * * *$ & $0.0235 *$ & $0.9897 *$ \\
\hline Twitter & 0.0742 & 0.6651 & $0.9913 * *$ \\
\hline
\end{tabular}

\subsection{Consensus Regions}

For policy purposes, it is useful to have a single set of regions to enable the implementation of stay-at-home orders and other mobility-related NPIs that are consistent with the severity of local 
outbreaks. Our method to obtain consensus regions (see Materials and Methods) results in 29 regions and a maximized modularity of $Q_{\max } \approx 0.92$ (see Figure 3 ). In these consensus regions, the state boundaries are often preserved; this is convenient administratively.

To allow policy makers to explore multiple scenarios for their communities, we have developed an online tool ${ }^{1}$ that creates on-the-fly regions for states, commuters, migration, and trip input data (because these networks produce the most effective COVID-19 regions among the ones that we explored). Users can change the weight of each of these input networks to customize regions, and they can download images of the regions and output data on county assignments.

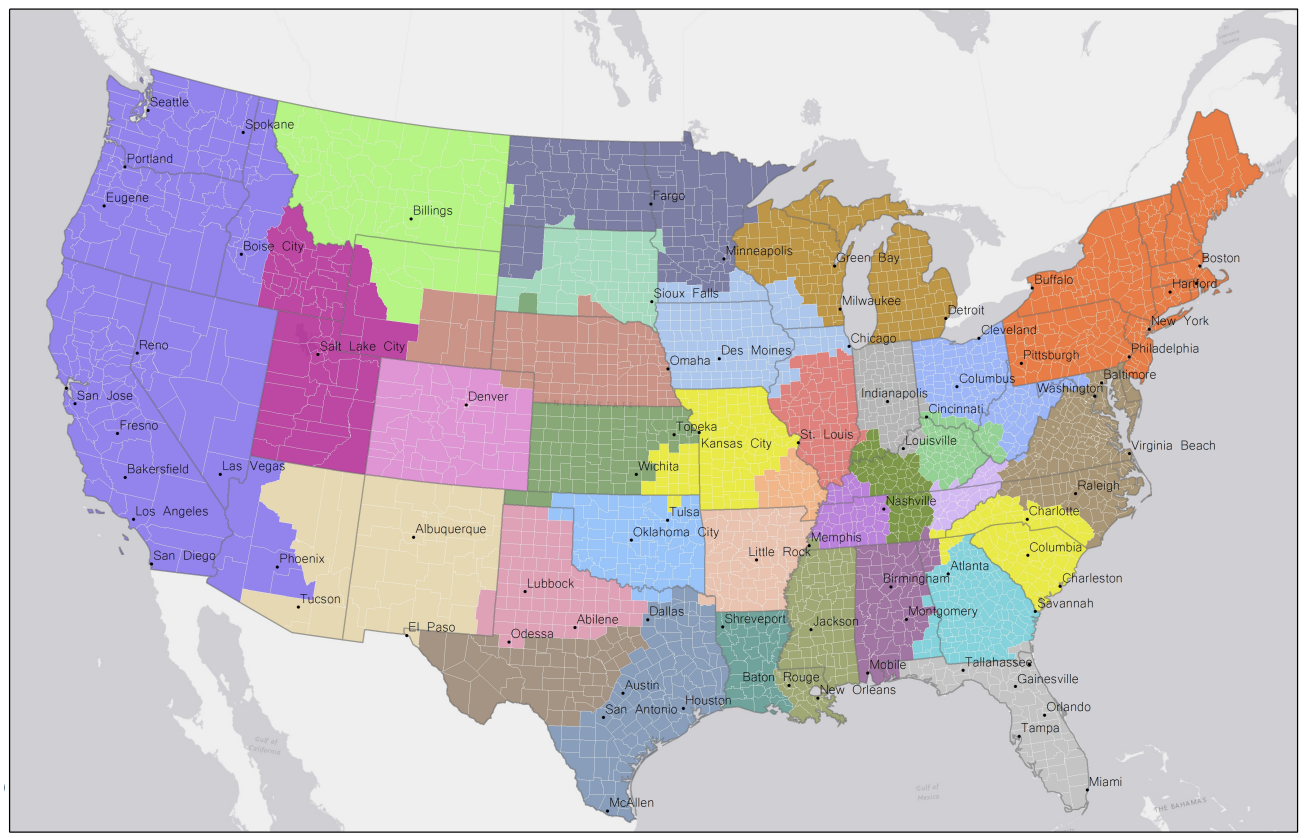

Figure 3: We construct consensus regions in the U.S. from an unweighted combination of the states and the regions that we obtained from each of the five human-behavior networks. These consensus regions represent areas of strong within-regional connectivity and weak between-regional connectivity.

\section{Conclusions and Discussion}

We evaluated how well the state boundaries and regions that we construct from human mobility and social networks capture natural boundaries in the geographic spread of COVID-19 infections over time. Although states are the predominant regions for administering policies for COVID-19 mitigation, we found that they are less effective than regions that we constructed from movement networks (specifically, commutes and trips) but more effective than regions that we constructed from social-network ties (specifically, Twitter and Facebook connections). These results likely occur because mobility regions are anchored by metropolitan areas; this yields strong connections in urban centers and suburbs and weaker connections in exurban areas. Consequently, these regions tend to have many cases of infection within regions and relatively few cases between regions. This finding reflects well-known regional-science principles that commuters and movers tend to follow an

\footnotetext{
${ }^{1}$ It is available at https://www.geo-social.com/consensus_regions/index.html.
} 
urban hierarchy with anchor cities and peripheries [24, 28, 19]. A regional approach is helpful for examining the spread of diseases (such as COVID-19) with scant geographic transmission statistics.

Based on our findings, we suggest that it is important to explore a set of consensus regions that are derived from human networks as ad-hoc administrative districts for making policy decisions for COVID-19 and other infectious diseases. Applying policies and messaging to county-based regions instead of states poses an inherent administrative burden that requires coordination and cooperative legislating. However, governors have created multi-state regions during the COVID19 pandemic $[44,33]$ and coalitions exist at the county level in economic development (e.g., the longstanding 420-county Appalachian Regional Commission [2]). Our regions may also be useful for examining the practicality of proposed inter-county alliances. To give one topical example, our modules do not resemble the recently proposed region of Greater Idaho [9]; instead, they illustrate that counties in Oregon have few existing connections to counties in Idaho. The U.S. federal government issues severe weather warnings (e.g., tornado, fire, storm, hurricane, and wind advisories) at the county level. State-level operations have also failed other health systems. For instance, the U.S. Organ Procurement and Transplantation Network recently implemented countylevel liver-transplant regions that are based on supply-and-demand optimization as an improvement over state-level regions [17].

Our work's shortcomings include a lack of up-to-date input data which was collected prior to the ongoing COVID-19 pandemic. Our mobility and social-media data predates the pandemic, so it may be misaligned with actual movement and information exchanges between counties. For instance, the migration data is from the period 2013-2017, Twitter data are from 2014-2015, and the mobility data is from January 2020. Additionally, conducting our research at the county level entails a mismatch in granularity across the U.S.; some counties have millions of individuals and are very large in area, whereas others have few people. Finally, because the spatial transmission of SARS-COV-2 is difficult to detect, we use rates in adjacent counties as a proxy for geographic transmission. However, we lack evidence that there were actual contagion events across these areas.

\section{Materials and Methods}

\subsection{Data Sets and Data Preparation}

We construct regions from five data sets that encode different types of human activity between the 3,108 counties in the Continental U.S.. We also use a county-adjacency network $G_{a}$ and associated COVID-19 case data. Conceptually, we can consider the five networks that we use to create the regions as independent variables. We treat the COVID-19 data on the $G_{a}$ network as an outcome variable.

\subsubsection{Movement and social-network data}

The 3,108 counties in our study area give the nodes of the networks. The edges in each of our five human-activity networks have edge weights that are based on the relevant activity. Each edge weight signifies some type of mobility or social-media connection (see Table 5). The Twitter and Facebook networks do not include all counties; in the data sets that we employed, some counties' populations do not have associated accounts or activity on these social networks.

The edge weight between counties $i$ and $j$ is the sum of the flows (for movement networks) or connections (for social-media networks) in both directions between the two counties. We allow self-edges, which we weight based on the number of connections whose origins and destinations are 
the same county. We obtain commuter data from the LODES ${ }^{2}$ residence-workplace characteristics (2015) from the U.S. Census [49]. Each flow represents a commute from home to work at the censusblock level. We obtain migration data from the American Community Survey (ACS) estimates of county-to-county migration flows for the period 2013-2017 [48]. Flows in this data set represent movers who change their county of residence from one year to the next. We use ACS migration data from a 5-year period to provide a large enough sample for estimates in small geographic areas. The flow estimates approximate the annual number of movers between counties for the 5 -year period of the data. We obtain GPS trace data (from January 2020) from SafeGraph [41]. In this data set, mobile phones are traced at the level of census-block groups and each trip is a flow from one block group to another (or is an internal trip within a block group). We use January 2020 because it is a recent month with a business-as-usual movement landscape.

We use Facebook's Social Connectedness Index (SCI), which is the number of Facebook friends between users in two counties divided by the product of the number of users in those counties [4]. The Twitter network encodes users with reciprocal mentions between 1 January 2014 and 31 December 2015. We obtained reciprocal user pairs from geolocated tweets that we collected using the Twitter Streaming API [47]. Although a follower-followee relationship does not imply an interpersonal tie between Twitter users, reciprocal mentions between two users indicate direct personal communication and possible interpersonal relationships [31]. See Table 6 for statistics about the correlations between the network data sets.

Table 5: Basic statistics of the network data sets

\begin{tabular}{lrrrr}
\hline Network & Date & Nodes $(n)$ & Edges $(e)$ & $\begin{array}{r}\text { Edge } \begin{array}{r}\text { Wum of } \\
\left(\times 10^{6}\right)\end{array} \\
\hline \text { Counties }\end{array} \quad 2018$ \\
Commutes & 2015 & 3108 & 8935 & $\mathrm{~N} / \mathrm{A}$ \\
Facebook & Undisclosed & 3108 & 211,404 & 284.9 \\
Migration & $2013-2017$ & 3108 & $9,625,506$ & $33,625.6$ \\
Trips & Jan. 2020 & 3108 & $3,313,328$ & 87.8 \\
Twitter & $2014-2015$ & 3085 & 535,424 & 2636.8 \\
\hline
\end{tabular}

\subsubsection{Similarities between input networks}

We measure the similarity between the five input networks by calculating the cosine similarity between networks (see Table 6). The networks are formatted as edge lists, where each row was a distinct edge in the network. The Facebook network has little overlap with the other four networks, so its edge weights differ from typical flows of inter-county information, mobility, and residential home-location movements (i.e., migration). However, the Twitter network follows commuting, migration, and trips flows. Therefore, some social-media data does appear to correlate with movement patterns.

\subsubsection{Assigning COVID-19 cases using a county-adjacency network}

We create a county-adjacency network $G_{a}$ in which the nodes are the 3,108 counties in the 48 contiguous states of the continental United States. We place each node at a county centroid. Each

\footnotetext{
${ }^{2}$ The acronym LODES stands for LEHD Origin-Destination Employment Statistics, and the acronym LEHD stands for Longitudinal Household Employer Dynamics.
} 
Table 6: Cosine similarity between the empirical networks

\begin{tabular}{lllll}
\hline & Facebook & Migration & Trips & Twitter \\
\hline Commutes & 0.025 & 0.977 & 0.955 & 0.941 \\
Facebook & & 0.025 & 0.031 & 0.015 \\
Migration & & & 0.969 & 0.939 \\
Trips & & & & 0.925 \\
\hline
\end{tabular}

edge is a line segment that connects geographically-adjacent counties (i.e., counties that share a boundary) that each had at least one COVID-19 case on record on or before 31 May 2020. There are 8,150 such edges.

We study COVID-19 cases between adjacent counties by calculating case counts C, case rates (per 1,000 people) $\mathrm{CR}$, and case-rate differences CD. We obtain the case numbers and case rates from The New York Times COVID-19 API [46], and we obtain the 2018 population data by county from the U.S. Center for Disease Control [8]. We assign COVID-19 case rates (CR) and case-rate differences (CD) to each edge in a network as follows. The mutual case rate of two counties is equal to the sum of case counts of the counties (i.e., nodes) multiplied by 1,000 and divided by the sum of their resident populations. The difference in case rates between two counties is equal to the absolute value of the case rate of one county minus the case rate of the other county. We put more credence into $\mathrm{CR}$ and $\mathrm{CD}$ values than into case counts because cases are population-dependent and to prevent overcounting. Given the nature of data on edges, we count COVID-19 cases multiple times when a node participates in multiple edges.

We consider three COVID-19 'waves' to assess the sensitivity of our calculations. These waves cover the dates 21 January 2020 - 31 May 2020 (wave 1), 1 June 2020 - 31 August (wave 2), and 1 September 2020 - 1 July 2021 (wave 3). These waves account for over 32.57 million cases. These dates correlate with peaks and troughs in COVID-19 case rates and death rates over time [12] that correspond to U.S. work and school activities.

\subsection{Analysis Methods}

\subsubsection{Regional delineation using community detection}

We detect communities in each of the five human-behavior networks. The numbers of communities are different for different community-detection methods. We use community detection to obtain hard partitions, so each county (i.e., each node) of a network can belong to only one community.

We measure the quality of our partitions from community detection by calculating the modularity objective function $[39,15]$ of these partitions. The modularity of a partition of a network is $Q=\sum_{i}\left(e_{i i}-b_{i}{ }^{2}\right)$, where $e_{i j}$ is the fraction of the total edge weight that connects communities $i$ and $j$ and $b_{i}$ is the fraction of all edge weight that is attached to community $i$.

One expects $Q$ to be large for a network partition in which there are few edges between the detected communities. We test five different algorithms for detecting communities in the networks. We use the Louvain locally greedy method for modularity maximization [5], an older greedy method for modularity maximization [11], InfoMap [40], and WalkTrap [38] using the IGRAPH package in the R Statistical Computing Environment. (In the software, the methods have the following names: cluster_louvain, cluster_fast_greedy, cluster_infomap, and cluster_walktrap, respectively). We also used the REDCAP algorithm, which partitions a network into communities using a spatial 
minimum spanning tree [22]. We implement REDCAP in PYTHON in the ARCGIS computing environment. We use results from the Louvain method in the main text, as they yielded the highest values of maximized modularity $Q_{\max }$. In SI data set S0, we give each county's community assignments for each of the five empirical networks using each of the five community-detection methods. We summarize these community-detection results in Table 7. Since the Louvain method [5] of maximizing modularity resulted in the largest values of $Q$ (see Table 1), we use this method to partition each of the five input networks.

Table 7: Summary of results of the community-detection algorithms

\begin{tabular}{llll} 
Method & Data Set & Number of Communities & Maximized Modularity $\left(Q_{\max }\right)$ \\
\hline Fast Greedy & Commutes & 30 & 0.902 \\
Fast Greedy & Facebook & 12 & 0.717 \\
Fast Greedy & Migration & 13 & 0.588 \\
Fast Greedy & Trips & 17 & 0.747 \\
Fast Greedy & Twitter & 12 & 0.531 \\
InfoMap & Commutes & 255 & 0.945 \\
InfoMap & Facebook & 8 & 0.865 \\
InfoMap & Migration & 49 & 0.795 \\
InfoMap & Trips & 134 & 0.910 \\
InfoMap & Twitter & 49 & 0.731 \\
Louvain & Commutes & 75 & 0.954 \\
Louvain & Facebook & 33 & 0.871 \\
Louvain & Migration & 29 & 0.801 \\
Louvain & Trips & 53 & 0.915 \\
Louvain & Twitter & 26 & 0.738 \\
REDCAP & Commutes & 48 & 0.898 \\
REDCAP & Facebook & 25 & 0.761 \\
REDCAP & Migration & 3 & 0.672 \\
REDCAP & Trips & 28 & 0.794 \\
REDCAP & Twitter & 28 & 0.608 \\
WalkTrap & Commutes & 1353 & 0.211 \\
WalkTrap & Facebook & 34 & 0.234 \\
WalkTrap & Migration & 96 & 0.245 \\
WalkTrap & Trips & 1 & 0.173 \\
WalkTrap & Twitter & 141 & 0.270 \\
\hline
\end{tabular}

\subsubsection{Resulting regions in the different networks}

After performing community detection, we construct geographic regions by assigning each county to a single community and eliminating interior boundaries between counties. Each region's territory naturally has one closed polygon.

In Figures 4-8, we show maps of the regions that we obtain using community detection on our five networks: commuters, Facebook relationships, migrants, trips, and Twitter co-mentions. Many of these networks tend to follow state boundaries; this is not by design, but instead occurs naturally in the data. When the regions do not follow state boundaries, natural features such as mountain 
ranges (e.g., the Appalachian range in the trip regions, as one can see in Figure 7) and infrastructure features such as highways (e.g., connections in Southern New Mexico and West Texas in the commuter regions, as one can see in Figure 4) can join regions across states or divide regions within states. Time zones may also play a role, as we see in the Facebook network in a division between the Central and Eastern time zones (see Figure 5).

For the Facebook, migration, and Twitter regions, we remove outlier counties, which look like "holes" in the map and arise from clusters of up to three counties. These counties occur mostly in the Great Plains and are likely not part of the surrounding communities because of their few connections (in the migration, Twitter co-mention, or Facebook friendship networks). The small number of connections results from their smaller populations or number of users. We reassign these counties to the neighboring region with the largest number of geographically-adjacent neighbors, with a preference for neighbors that are in the same state. (There were no instances in which a county shares a border with an equal number of counties from two different communities.)

\subsubsection{Similarities between regions}

We assess the similarities of different county assignments to various regions by calculating Jaccard indices and z-scores of Rand coefficients (Tables 9 and 8).

Table 8: Similarities between the sets of regions using the z-Score $z_{R}$ of the Rand coefficient (The values in the table are $z_{R} \times 10^{-5}$ )

\begin{tabular}{llllll}
\hline & Facebook & Migration & States & Trips & Twitter \\
\hline Commutes & 2.656 & 3.331 & 2.691 & 3.329 & 3.290 \\
Facebook & & 6.227 & 5.200 & 3.784 & 6.230 \\
Migration & & & 7.385 & 5.419 & 10.758 \\
States & & & & 3.991 & 7.680 \\
Trips & & & & & 5.118 \\
\hline
\end{tabular}

Table 9: Similarities between the sets of regions using the Jaccard index of similarity

\begin{tabular}{llllll}
\hline & Commutes & Facebook & Migration & Trips & Twitter \\
\hline & & & & & \\
Facebook & 0.761 & & & & \\
Migration & 0.791 & 0.862 & & & \\
Trips & 0.845 & 0.801 & 0.843 & & \\
Twitter & 0.769 & 0.828 & 0.892 & 0.808 & \\
States & 0.771 & 0.866 & 0.905 & 0.820 & 0.880 \\
\hline
\end{tabular}

\subsubsection{Permutation tests}

In one permutation test, we shuffle the edge labels (i.e., whether they are within-region edges or between-region edges) uniformly at random. We then sum the case values (either C, CR, or CW) over only between-region edges. We run this test 10,000 times and thereby produce a distribution of expected sums for between-region crossings. We compare this distribution to the sum of case 
values of actual between-region crossings. We perform the test separately for each of of the three types of case values and for each region type.

\subsubsection{Creating consensus regions}

We combine the states and each of the five sets of regions from empirical data to create a single set of consensus regions. We weight each pair of counties in the network $G_{a}$ by the number of times that both counties appear in the same region (or state, for the state network), so edge weights range between 0 and 6 . We create consensus regions by applying the Louvain modularity-maximization algorithm to this agreement network (see Figure 9).

To create consensus regions, we calculate the amount of agreement between edges in an adjacency matrix between counties. We assign an edge weight between adjacent counties that indicates the number of times that the pair of counties is assigned to the same community (or is in the same state). The values of the edge weights range from 0 (i.e., never in the same community or state) to 6 (i.e., always in the same community or state).

By visualizing these numbers in a map, we see the locations of strong and weak agreement (see Figure 9). We then apply the Louvain community-detection algorithm to this weighted adjacency network to produce consensus regions. We use the concept of border 'thickness', where thicker borders are ones that that separate communities more times [29]. (In our study, the maximum number of times is six.) Thick borders indicate that there are relatively few crossings and connections across geographic space.

\section{Acknowledgements}

MAP acknowledges support from the National Science Foundation (grant number DMS-2027438) through the RAPID program. CA acknowledges support from the National Science Foundation (grant number HNDS-R-2045271). We thank Geng Tian for developing our Web application. We thank SafeGraph and Facebook for providing data. CA thanks the Geospatial Fellows Program at the University of Illinois. We thank Grant D. Brown and William Drummond for helpful comments.

\section{References}

[1] B. M. Althouse, B. Wallace, B. Case, S. V. Scarpino, A. Allard, A. M. Berdahl, E. R. White, and L. Hébert-Dufresne. The unintended consequences of inconsistent pandemic control policies, 2020. MedRxiv, Available at https://doi.org/10.1101/2020.08.21.20179473.

[2] Appalachian Regional Commission. About the appalachian regional commission, n.d. Available at https://www.arc.gov/about-the-appalachian-regional-commission/ (accessed 12 July 2021).

[3] J. Arino. Describing, modelling and forecasting the spatial and temporal spread of COVID-19: A short review, 2021. ArXiv:2102.02457.

[4] M. Bailey, R. Cao, T. Kuchler, J. Stroebel, and A. Wong. Social connectedness: Measurement, determinants, and effects. Journal of Economic Perspectives, 32(3):259-280, 2018.

[5] V. D. Blondel, J.-L. Guillaume, R. Lambiotte, and E. Lefebvre. Fast unfolding of communities in large networks. Journal of Statistical Mechanics: Theory and Experiment, 2008(10):P10008, 2008 . 


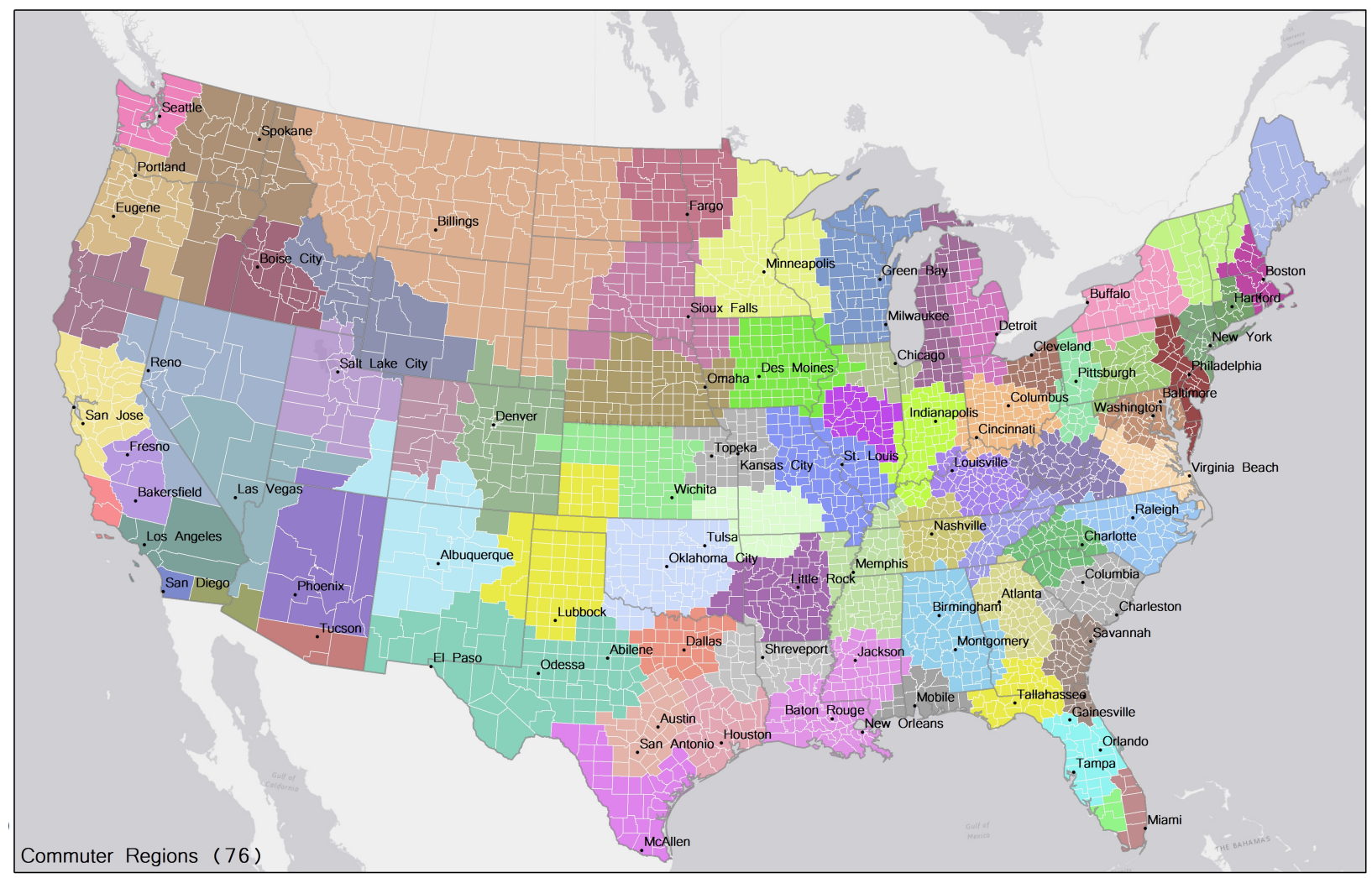

Figure 4: Regions that we construct using a network of commuters from the LODES U.S. Census data [49] that is aggregated to the county level from census blocks. (Block groups are an areal unit at which the U.S. Census provides demographic data.) This yields 76 regions, which is the most regions of any network that we examine. 


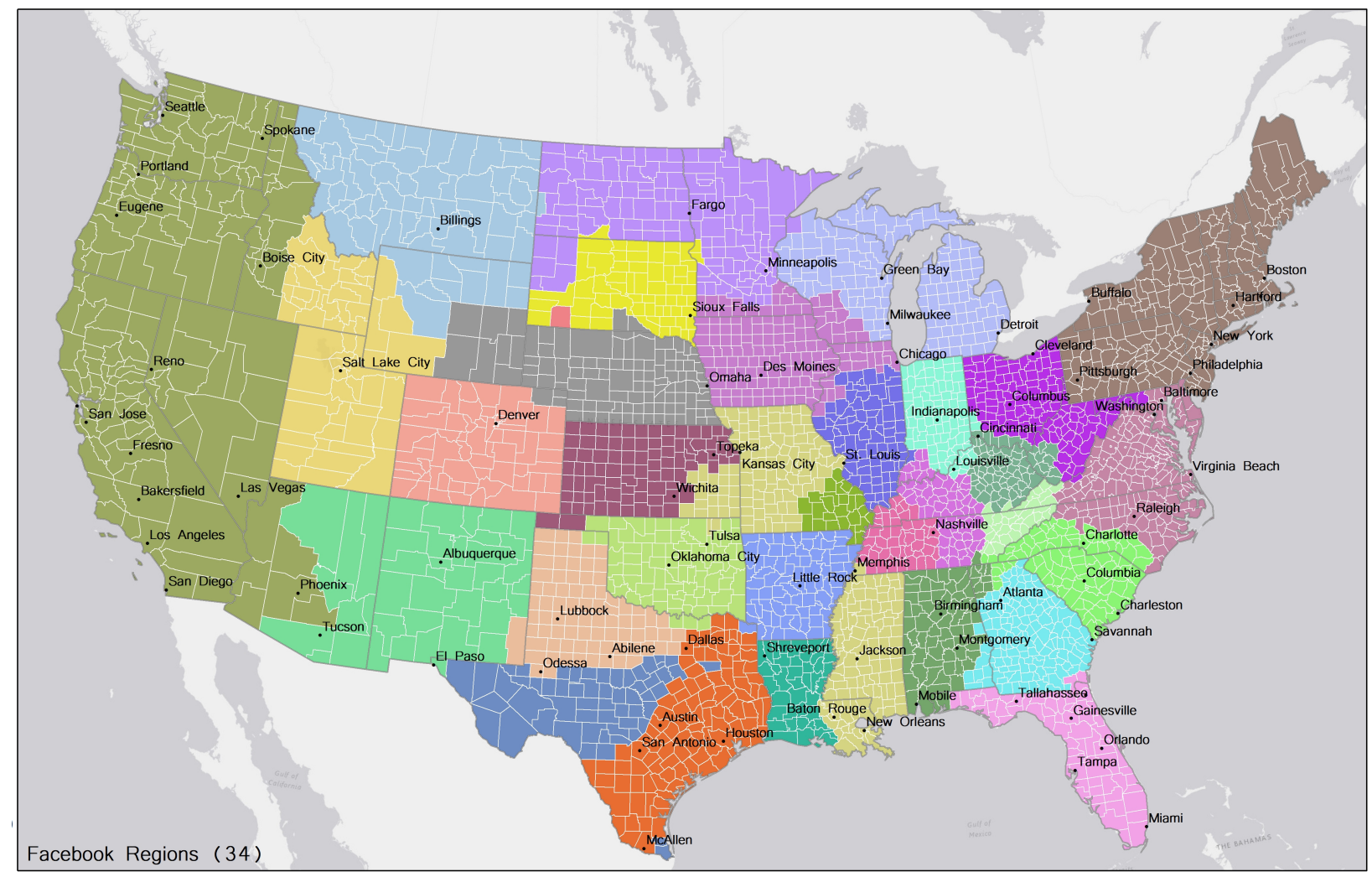

Figure 5: Regions that we construct from Facebook friendships using the Facebook Social Connectivity Index (SCI) [4], which is the number of Facebook friendships between two counties divided by the total number of Facebook users in those two counties. We reassign two counties in Texas to the community of their surrounding counties (i.e., those with which the focal county shares a border) because we require communities to be geographically contiguous. There are a total of 34 regions, including one very large region that includes the entire West Coast of the U.S. 


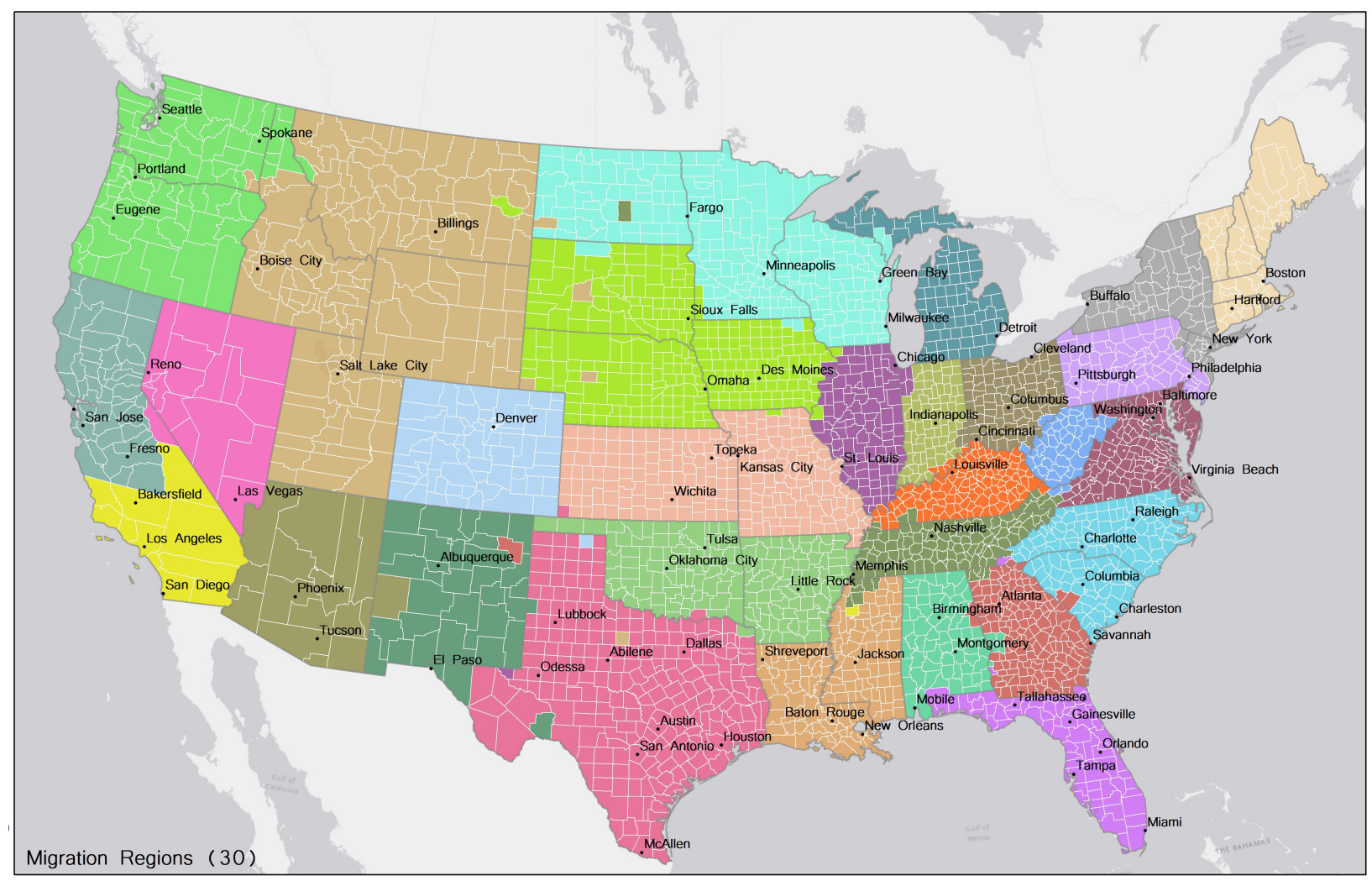

Figure 6: Regions that we construct using a migration network from the U.S. American Community Survey (ACS) [48]. There are a total of 30 regions. In the migration network, outliers often belong to a distant community. (For example, a county in Michigan can be part of a community that is based in Florida because the nature of inter-metropolitan migration.) 


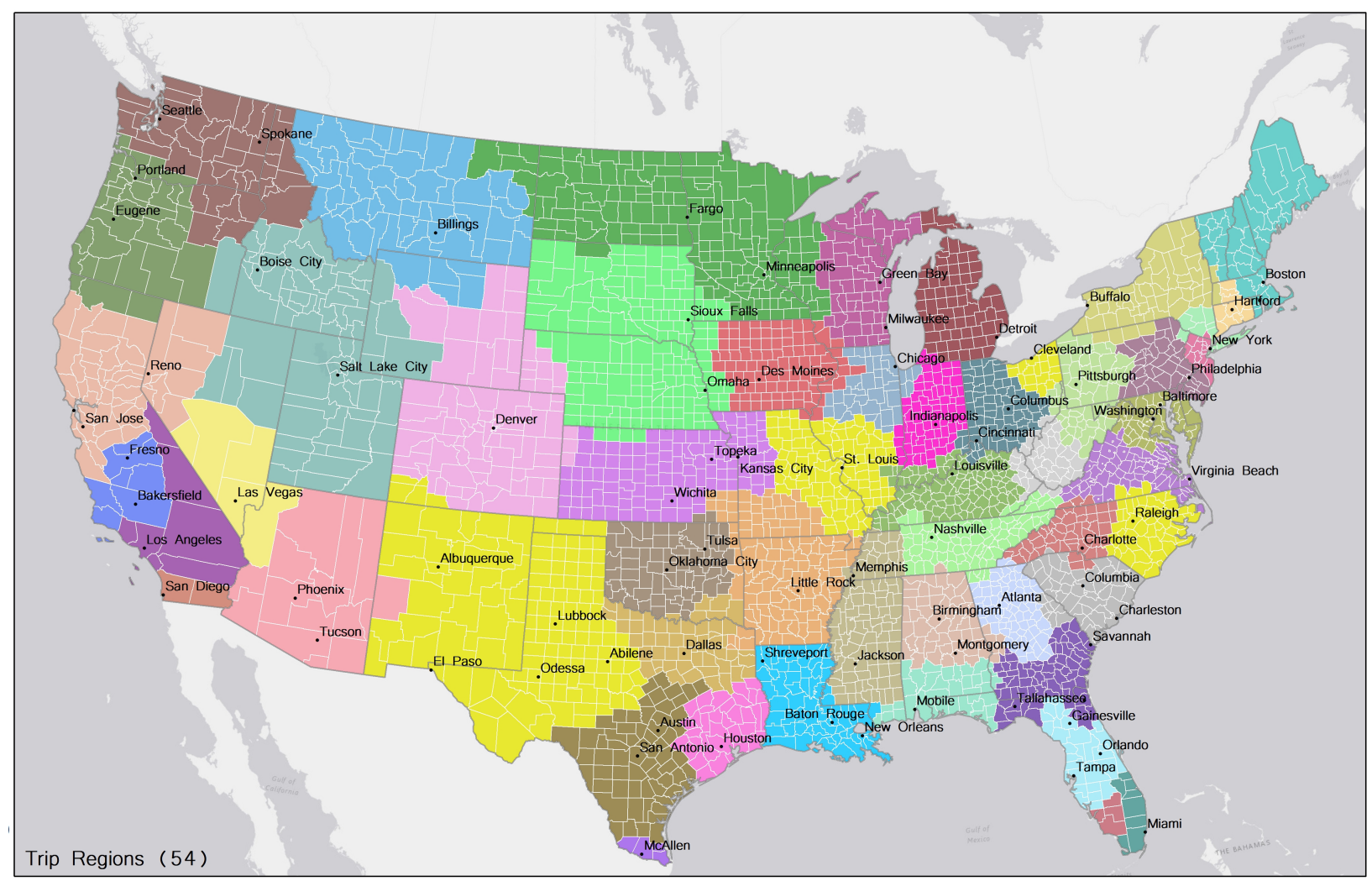

Figure 7: Regions that we construct using GPS traces (i.e., trips) from SafeGraph [41] in January 2020. We aggregate the data from the block-group level to the county level. There are a total of 76 regions. 


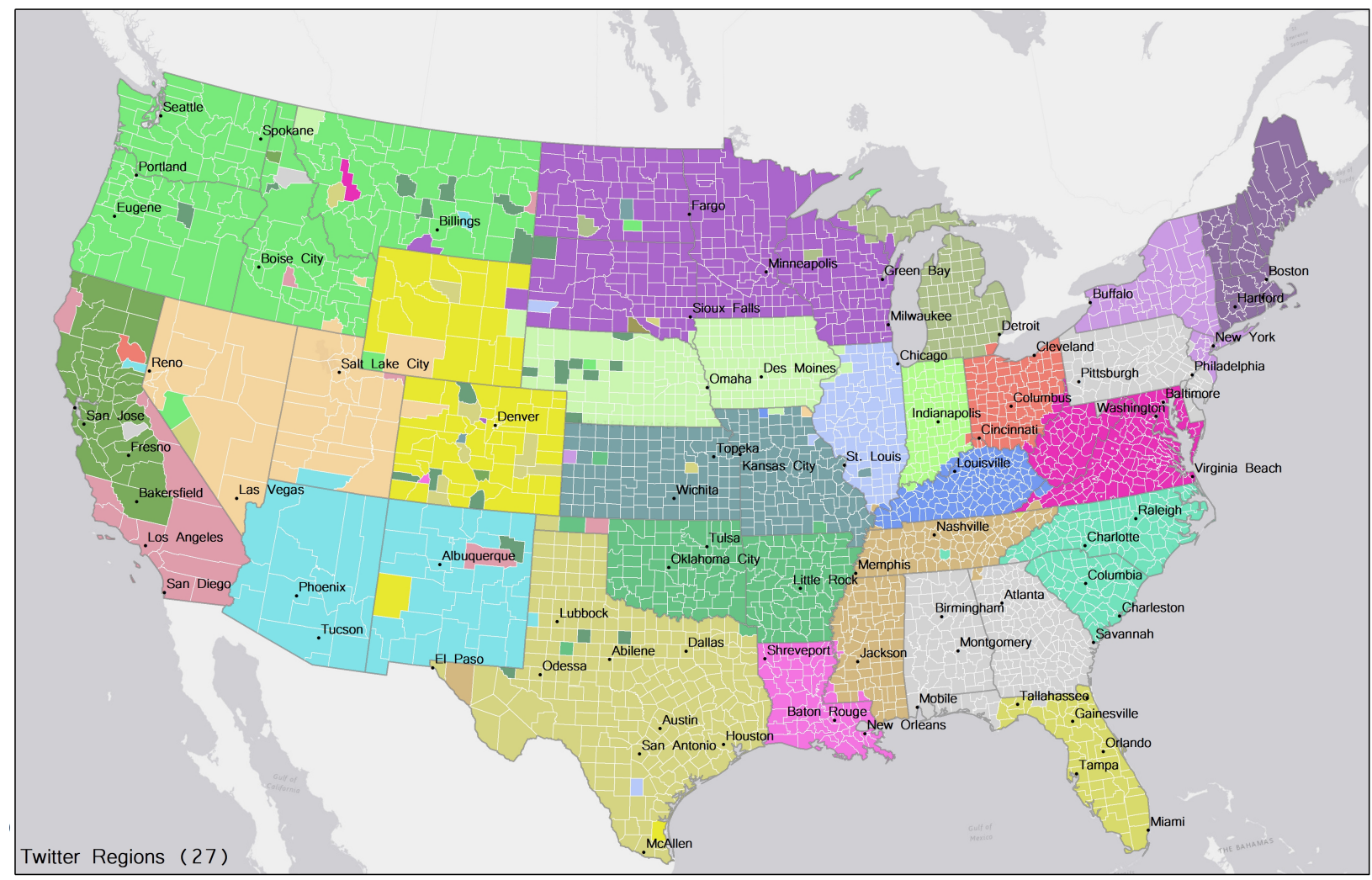

Figure 8: Output for regions that we construct from a network of Twitter co-mentions from [47]. There are a total of 27 regions. Counties that do not match their surrounding communities likely arise from a low population and accordingly few Twitter users. 


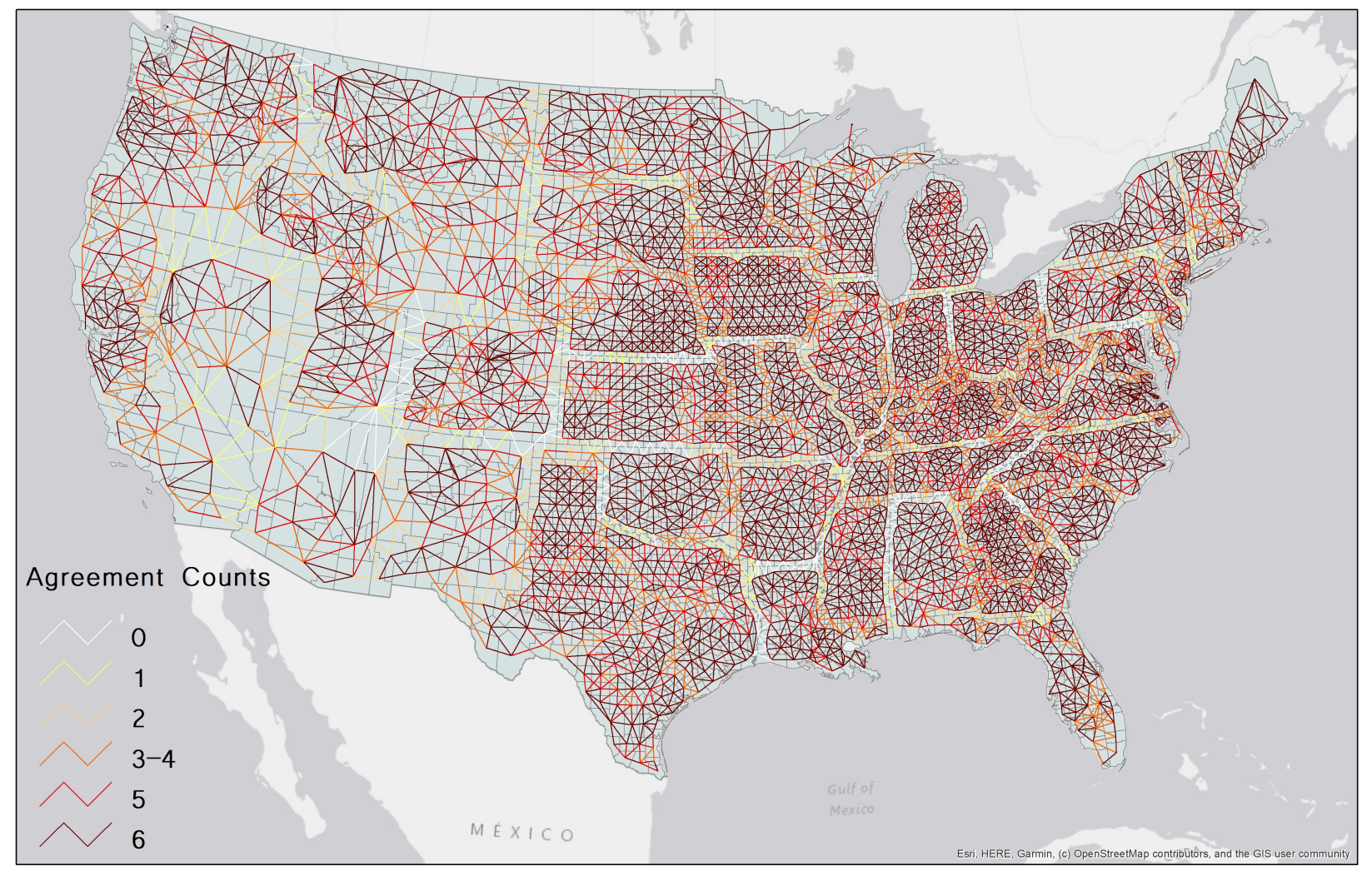

Figure 9: The agreement count between adjacent counties (i.e., counties that share a boundary). We weight the edges of this network by the number of times that the edge's nodes are placed into the same region or state, with 6 possible opportunities to agree. The visualization illustrates natural divisions between states and regions in the U.S. 
[6] C. Brelsford, G. Thakur, R. Arthur, and H. Williams. Using digital trace data to identify regions and cities. In Proceedings of the 2nd ACM SIGSPATIAL International Workshop on Advances on Resilient and Intelligent Cities, pages 5-8, 2019.

[7] S. R. Brooker-Gross. News and metropolitan hinterland and hierarchy. Urban Geography, $4(2): 138-155,1983$.

[8] Centers for Disease Control and Prevention. United States COVID-19 Cases and Deaths by State, n.d. Available at https://covid.cdc.gov/covid-data-tracker/ (accessed 10 July 2021).

[9] B. Chapell. Oregone? 7 Oregon counties vote to back seceding, so citizens can vote GOP in Idaho, 2021. National Public Radio, https://www.npr.org/2021/05/20/998660102/ oregone-7-oregon-counties-vote-to-back-seceding-so-citizens-can-vote-gop-in -idah.

[10] W. A. Chiu, R. Fischer, and M. L. Ndeffo-Mbah. State-level needs for social distancing and contact tracing to contain COVID-19 in the United States. Nature Human Behaviour, 4(10):1080-1090, 2020.

[11] A. Clauset, M. E. J. Newman, and C. Moore. Finding community structure in very large networks. Physical review E, 70(6):066111, 2004.

[12] J. Drake. The real cause of America's third wave of COVID-19, 2020. Forbes Magazine, Available at https://www.forbes.com/sites/johndrake/2020/12/07/ the-real-cause-of-americas-third-wave-of-covid-19/?sh=1217988812fd.

[13] C. Ducruet and L. Beauguitte. Spatial Science and Network Science: Review and Outcomes of a Complex Relationship. Networks and Spatial Economics, 14(3-4):297-316, 2014.

[14] L. Ferretti, C. Wymant, M. Kendall, L. Zhao, A. Nurtay, L. Abeler-Dörner, M. Parker, D. Bonsall, and C. Fraser. Quantifying SARS-CoV-2 transmission suggests epidemic control with digital contact tracing. Science, 368(6491):eabb6936, 2020.

[15] S. Fortunato and D. Hric. Community detection in networks: A user guide. Physics Reports, 659:1-44, 2016.

[16] S. Foster. As COVID-19 proliferates mayors take response lead, sometimes in conflict with their governors, 2020. Georgetown Law SALPAL, Available at https://www.law.georgetown. edu/salpal/as-covid-19-proliferates-mayors-take-response-lead-sometimes-in -conflicts-with-their-governors/.

[17] S. Gentry, E. Chow, A. Massie, and D. Segev. Gerrymandering for justice: Redistricting Us liver allocation. Interfaces, 45(5):462-480, 2015.

[18] H. L. Green. Hinterland boundaries of New York City and Boston in Southern New England. Economic Geography, 31(4):283-300, 1955.

[19] M. J. Greenwood. Human migration: theory, models, and empirical studies. Journal of Regional Science, 25(4):521-544, 1985.

[20] R. Guimera, S. Mossa, A. Turtschi, and L. A. N. Amaral. The worldwide air transportation network: Anomalous centrality, community structure, and cities' global roles. Proceedings of the National Academy of Sciences of the United States of America, 102(22):7794-7799, 2005. 
[21] J.-M. Guldmann. Spatial interaction models of international telecommunication flows. In M. Goodchild and D. Jannelle, editors, Best Practices in Spatially Integrated Social Science, pages 400-442. Oxford University Press, Oxford, UK, 2004.

$[22]$ D. Guo. Regionalization with dynamically constrained agglomerative clustering and partitioning (REDCAP). International Journal of Geographical Information Science, 22(7):801-823, 2008 .

[23] J. Hadfield, C. Megill, S. M. Bell, J. Huddleston, B. Potter, C. Callender, P. Sagulenko, T. Bedford, and R. A. Neher. Nextstrain: Real-time tracking of pathogen evolution. Bioinformatics, 34(23):4121-4123, 2018.

[24] P. Haggett and R. J. Chorley. Network Analysis in Geography. Edward Arnold, London, UK, 1969.

[25] S. Hazarie, D. Soriano-Panos, A. Arenas, J. Gómez-Gardeñes, and G. Ghoshal. Interplay between intra-urban population density and mobility in determining the spread of epidemics, 2021. ArXiv:2102.00671 (10 Feb 2021).

[26] D. Holtz, M. Zhao, S. G. Benzell, C. Y. Cao, M. A. Rahimian, J. Yang, J. Allen, A. Collis, A. Moehring, T. Sowrirajan, et al. Interdependence and the cost of uncoordinated responses to COVID-19. Proceedings of the National Academy of Sciences of the United States of America, 117(33):19837-19843, 2020.

[27] X. Hou, S. Gao, Q. Li, Y. Kang, N. Chen, K. Chen, J. Rao, J. S. Ellenberg, and J. A. Patz. Intracounty modeling of covid-19 infection with human mobility: Assessing spatial heterogeneity with business traffic, age, and race. Proceedings of the National Academy of Sciences, 118(24), 2021.

[28] W. Isard. Regional science, the concept of region, and regional structure. Papers in Regional Science, 2(1):13-26, 1956.

[29] M. Jin, L. Gong, Y. Cao, P. Zhang, Y. Gong, and Y. Liu. Identifying borders of activity spaces and quantifying border effects on intra-urban travel through spatial interaction network. Computers, Environment and Urban Systems, 87:101625, 2021.

[30] C. F. Kohn. Regions and regionalizing. Journal of Geography, 69(3):134-140, 1970.

[31] C. Koylu. Discovering multi-scale community structures from the interpersonal communication network on Twitter. In L. Perez, E.-K. Kim, and R. Sengupta, editors, Agent-Based Models and Complexity Science in the Age of Geospatial Big Data, pages 87-102. Springer International Publishing, Cham, Switzerland, 2018.

[32] X. Liu, R. Hollister, and C. Andris. Wealthy hubs and poor chains: Constellations in the U.S. urban migration system. In Agent-Based Models and Complexity Science in the Age of Geospatial Big Data, pages 73-86. Springer-Verlag, Heidelberg, Germany, 2018.

[33] T. Luna. California, Oregon and Washington to work together on plan to lift coronavirus restrictions, 2020. Available at https://www.latimes.com/california/story/ 2020-04-13/coronavirus-restrictions-gavin-newsom-california-washington-oregon -western-state-pact.

[34] I. Masser and J. Scheurwater. Functional regionalisation of spatial interaction data: an evaluation of some suggested strategies. Environment and Planning A, 12(12):1357-1382, 1980. 
[35] A. C. Miller, N. J. Foti, J. A. Lewnard, N. P. Jewell, C. Guestrin, and E. B. Fox. Mobility trends provide a leading indicator of changes in SARS-CoV-2 transmission, 2020. medRxiv, Available at doi.org/10.1101/2020.05.07.20094441.

[36] V. T. Noronha and M. F. Goodchild. Modeling interregional interaction: Implications for defining functional regions. Annals of the Association of American Geographers, 82(1):86-102, 1992.

[37] A. K. Philbrick. Principles of areal functional organization in regional human geography. Economic Geography, 33(4):299-336, 1957.

[38] P. Pons and M. Latapy. Computing communities in large networks using random walks. In International Symposium on Computer and Information Sciences, pages 284-293. Springer, 2005 .

[39] M. A. Porter, J. Onnela, and P. J. Mucha. Communities in networks. Notices of the American Mathematical Society, 56(9):1082-1097, 1164-1166, 2009.

[40] M. Rosvall and C. T. Bergstrom. Maps of random walks on complex networks reveal community structure. Proceedings of the National Academy of Sciences, 105(4):1118-1123, 2008.

[41] SafeGraph. COVID-19 Data Consortium, 2020. Available at https://www.safegraph.com/ academics (accessed 5 May 2021).

[42] F. Schlosser, B. F. Maier, O. Jack, D. Hinrichs, A. Zachariae, and D. Brockmann. COVID-19 lockdown induces disease-mitigating structural changes in mobility networks. Proceedings of the National Academy of Sciences of the United States of America, 117(52):32883-32890, 2020.

[43] C. Seto, A. Khademi, C. Graif, and V. G. Honavar. Commuting network spillovers and COVID-19 deaths across US counties, 2021. ArXiv:2010.01101 (10 Feb 2021).

[44] K. Sgueglia and C. Kelly. Seven Midwestern governors announce their states will coordinate on reopening, 2020. Available at https://www.cnn.com/2020/04/16/politics/ midwest-governors-reopening-pact/index.html.

[45] Y. Takhteyev, A. Gruzd, and B. Wellman. Geography of Twitter networks. Social Networks, $34(1): 73-81,2012$.

[46] The New York Times. Coronavirus in the U.S.: Latest map and case count., 2020. Available at https://www.nytimes.com/interactive/2020/us/coronavirus-us-cases.html (accessed 15 April 2021).

[47] Twitter, Inc. Twitter Streaming API, 2021. Available at https://developer.twitter.com/ en/products/twitter-api (accessed 15 December 2020).

[48] U.S. Census Bureau. 2013-2017 American Community Survey Migration/Geographic Mobility Data, 2020. Available at https://www.census.gov/topics/population/migration/data/ tables/acs.2017.html (accessed 15 April 2021).

[49] U.S. Census Bureau. Longitudinal Household Employer Dynamics LEHD-LODES residenceworkplace characteristics, 2020. Available at https://lehd.ces.census.gov/data (accessed 15 December 2020).

[50] C. Xiong, S. Hu, M. Yang, W. Luo, and L. Zhang. Mobile device data reveal the dynamics in a positive relationship between human mobility and COVID-19 infections. Proceedings of the National Academy of Sciences of the United States of America, 117(44):27087-27089, 2020. 